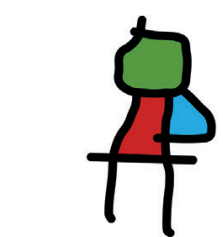

SEICAIP

\section{Allergologia et immunopathologia}

Sociedad Española de Inmunología Clínica, Alergología y Asma Pediátrica

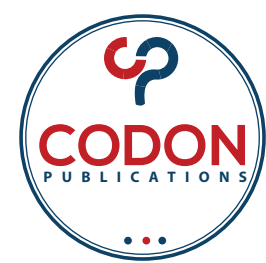

RESEARCH ARTICLE

\title{
MEF 2C alleviates acute lung injury in cecal ligation and puncture (CLP)-induced sepsis rats by up-regulating AQP1
}

\author{
Wenmei Liang ${ }^{\mathrm{a}}$, Li Guo ${ }^{\mathrm{b}}$, Tonghua Liua , Song Qina* \\ ${ }^{a}$ Department of Critical Care Medicine, The Affiliated Hospital of Zunyi Medical University, Zunyi, Guizhou 563000, China \\ ${ }^{b}$ Department of Critical Care Medicine, Zhongshan Torch Development Zone Hospital, Zhongshan, Guangdong 528437, China
}

Received 7 May 2021; Accepted 12 August 2021

Available online 1 September 2021

\section{KEYWORDS}

apoptosis;

AQP1;

inflammation;

MEF2C;

sepsis

\begin{abstract}
Background: Sepsis is a systemic inflammatory response syndrome and leads to patient's death. Objective: To investigate the effect of myocyte enhancer factor 2 (MEF2C) on acute lung injury (ALI) with sepsis and its possible mechanism.

Material and Methods: The cecal ligation and puncture (CLP)-induced sepsis rat model was established. The lung injury was determined by lung wet-dry weight ratio, the concentration of inflammatory cytokines, including tumor necrosis factor- $\alpha$ (TNF- $\alpha$ ), Interlukin (IL)-6, IL-1 $\beta$, and IL-10, were measured by the enzyme-linked-immunosorbent serologic assay kit. The cell apoptosis was detected by TUNEL staining assay.

Results: Interestingly, MEF2C was down-regulated in this model. Moreover, adeno-associated virus (AAV)-MEF2C treatment markedly suppressed TNF- $\alpha$, IL-1 $1 \beta$, and IL- 6 concentrations but promoted IL-10 concentration in serum in CLP-challenged rats. Besides, overexpression of MEF2C alleviates CLP-induced lung injury. Interestingly, AAV-MEF2C treatment was confirmed to suppress apoptosis in CLP-induced sepsis rats as well as promote aquaporin APQ1 expression. Mechanistically, the rescue experiments indicated that MEF2C alleviated CLP-induced lung inflammatory response and apoptosis via up-regulating AQP1.

Conclusion: In summary, overexpression of MEF2C suppressed CLP-induced lung inflammatory response and apoptosis via up-regulating AQP1, providing a novel therapeutic target for sepsis-induced ALI.

๑) 2021 Codon Publications. Published by Codon Publications.
\end{abstract}

*Corresponding author: Song Qin, Department of Critical Care Medicine, The Affiliated Hospital of Zunyi Medical University, No. 149, Dalian Road, Zunyi, Guizhou 563000, China. E-mail address: sqin5233@163.com 


\section{Introduction}

Sepsis is a systemic inflammatory response syndrome that affects multiple systems and organs, even leading to patient's death. ${ }^{1,2}$ In sepsis, various inflammatory factors and inflammatory mediators, including tumor necrosis factor $\alpha$ (TNF- $\alpha$ ), interleukin (IL)-6, and IL-10 are released and activated, resulting in pro- and anti-inflammatory processes, which means the immune function of the body is damaged. Among them, acute lung and kidney injuries are common and serious complications of sepsis patients, with an incidence rate of as high as $40-70 \%$. In the majority of cases $(>70 \%)$, sepsis seriously threatens the safety of patients. ${ }^{3,4}$ Acute lung injury (ALI) is one of the most common fatal complications of sepsis due to lung susceptibility. Although numerous studies have investigated the pathogenesis and diagnosis of sepsis-induced ALI, the current treatment options are limited. Therefore, finding novel and more effective therapeutic drugs would contribute to the treatment of sepsis-induced ALI. ${ }^{5}$

The myocyte enhancer factor 2 (MEF2) family of transcription factors consists of MEF2A, B, C, and D members. A recent study has shown that MEF2 is associated with inflammatory diseases, in particular, limitation of MEF2C can promote the expression of pro-inflammatory chemokines (CCL2 and CCL5) and cytokines (IL-1 $\beta$ and TNF). ${ }^{6}$ Moreover, overexpression of MEF2C inhibits induction of pro-inflammatory molecules by TNF- $\alpha$, activation of nuclear factor kappa-light-chain-enhancer of activated $B$ cells $(N F-\kappa B)$, and adhesion of leukocytes to endothelial cells. ${ }^{7}$ In addition, MEF2 is normally expressed in lung tissues, especially MEF2C is down-regulated in pulmonary hypertension. ${ }^{8}$ However, the role of MEF2C in sepsis-induced ALI still remains unclear.

Aquaporins (AQPs) are a small and complete family of membrane proteins that facilitate water transport and small neutral solutes across various biofilms in various eukaryotes and prokaryotes. ${ }^{9}$ Aquaporin AQP-1 is expressed in pulmonary endothelial cells and regulates water transport and plays an important role in many pulmonary diseases. ${ }^{10}$ Notably, both mRNA and protein levels of AQP-1 are significantly down-regulated in ALI rats, whereas up-regulation of AQP1 can protect the lungs from injury.11 Interestingly, overexpression of MEF2C increases the AQP1 mRNA and protein levels in human umbilical cord vein endothelial (HUVEC) cells. $^{12}$ Nevertheless, the association of MEF2C and AQP1 in sepsis-induced ALI is unknown.

In this study, we established cecal ligation and puncture (CLP)-induced sepsis rat model, and investigated the function of MEF2C on inflammatory response and apoptosis in lung tissues of rats, which might be mediated by AQP1 expression. Our results suggested the importance of a MEF2C-AQP1 axis in sepsis-induced ALI and provided mechanistic insight into how MEF2C is associated with sepsis.

\section{Method and materials}

The animal experiments were conducted in accordance with the Guide for the Care and Use of Laboratory Animals. Ethical approval was obtained from the Ethics Committee of the Affiliated Hospital of Zunyi Medical University.

\section{Animals}

Male Sprague-Dawley (SD) rats $(\mathrm{n}=48)$, weighing $200 \pm 20 \mathrm{~g}$, were purchased from Nanjing Zunke (China). Rats were housed at a room temperature of $21 \pm 2^{\circ} \mathrm{C}$, humidity of $55-65 \%$, and 12 -h light/12-h dark conditions, and received adaptive feeding for 1 week. The rats were randomly divided into the following four groups: Sham, CLP, CLP+adeno-associated virus (AAV)-shRNA (Scramble) (Hanheng Company, China), and CLP+AAV-MEF2C (Hanheng Company, China).

The recombinant AAV containing MEF2C was purchased from Hanheng Company (China). The AAV expressing no transgene was used as negative control (AAV-Scramble). Three weeks before construction of CLP model, the rats were anesthetized and randomized to intratracheal instillation of surfactant-phosphate buffer solution (PBS) mixture containing (a) $3.5 \times 10^{10} \mathrm{drp}$ AAV-Scramble, or (b) $3.5 \times 10^{10}$ drp AAV-MEF2C. The efficacy of overexpressed MEF2C was evaluated by western blot test.

Two days before CLP treatment, the rats received intraperitoneal administration of $1 \%$ pentobarbital sodium $(80 \mathrm{mg} / \mathrm{kg})$, and were injected with intravenous sh-AQP1 (Genepharma, China) or scrambled shRNA (NC) at a dose of $4 \times 10^{7}$ transduction units $/ \mathrm{mL}$.

The CLP model was used to induce sepsis and established by intraperitoneal injection of pentobarbital sodium $(10 \mathrm{mg} /$ $\mathrm{kg}$ body wt.). A 2-3-cm incision along the midline of the abdomen, at the end of the cecum was positioned, the cecum was punctured with a \#20 needle, and the proximal end of the cecum was ligated and closed. The Sham rats underwent the same operation, except CLP. After 12 and $24 \mathrm{~h}$, the rats were anesthetized with $5 \%$ isoflurane, and euthanized by cervical dislocation, the left lung was isolated, and blood was washed with pre-cooled PBS, respectively.

\section{Hematoxylin and eosin (H\&E) staining}

The lung tissues were dissected, washed, and fixed with $4 \%$ paraformaldehyde. These were embedded in paraffin, sectioned at 4- $\mu \mathrm{m}$ thickness, de-waxed and rehydrated, and stained with H\&E straining kit (Solarbio, China). The histologic injury scores were calculated by the sum of the score for pulmonary edema (PE), inflammatory cell infiltration, alveolar hemorrhage, hyaline membrane, and atelectasis. Each histologic characteristic was evaluated on a scale from 0 to 5: 0: normal (no injury); 1: minimal, injured area $\leq 25 \%$; 2: mild, $26-50 \%$ injured area; 3: moderate, $51-75 \%$ injured area; 4 : severe, $76-90 \%$ injured area; and 5: extremely severe, injured area $>90 \%$. Three regions were randomly selected for each slide and their averages were used as a histopathologic score.

\section{Enzyme-linked-immunosorbent serologic assay (ELISA)}

Levels of tumor necrosis factor (TNF)- $\alpha$, interleukin (IL)-6, IL-1 $\beta$, and IL-10 were assessed using commercial ELISA kits (Elabscience Biotechnology Co. Ltd., China) according to the manuscript's protocol. 


\section{Myeloperoxidase (MPO) activity assay}

MPO activity was measured by an MDA detection kit (Nanjing Jiancheng Bioengineering Institute, China) after homogenization of the lung tissue.

\section{Terminal deoxynucleotidyl transferase-mediated dUTP nick end labeling (TUNEL) assay}

The lung tissues were fixed in polyformaldehyde, and dehydrated and embedded in paraffin. The TUNEL staining procedure (Millipore, Germany) was strictly followed to detect the lung tissue apoptosis. Staining of the lung tissue was visualized under light microscope, which showed red staining of positive apoptotic cells. Approximately 10 visual fields (high magnification $\times 400$ ) were selected.

\section{Reverse transcription-quantitative polymerase chain reaction (RT-qPCR)}

Total RNA from the lung tissues was extracted with Trizol (Invitrogen, USA) and complementary DNA (CDNA) was synthesized with RNA transcription kit (Promega, USA). Then RT-qPCR was performed with SYBR Green qRTPCR kit (Takara, Japan). The primer sequences are as follows: MEF2C, forward: 5'-CTTCTGCCACTGGCCCAC-3', reverse: 5'-GGGGTTGCCGTATCCATTC-3'; AQP1, forward: 5'-GCTGTGGTGGCTGAGTTCC-3', reverse: 5'-TGTGACCGCTGG GTTGAG-3'; and GAPDH, forward: 5'-CTGGAGAAACCTGCCAA GTATG-3', reverse: 5'-GGTGGAAGAATGGGAGTTGCT-3'.

\section{Western blot}

Total protein from the lung tissues was extracted using RIPA lysis buffer, and protein concentration was determined using a BCA protein kit (Beyotime, China). Protein samples $(30 \mu \mathrm{g})$ were separated with $10 \%$ SDS-PAGE, and transferred onto polyvinylidene difluoride (PVDF) membranes. The membranes were blocked with one of the primary antibodies at $4^{\circ} \mathrm{C}$ overnight, including $\mathrm{BAX}(1 / 800$; Abcam, UK), Bcl-2 (1/800; Abcam, UK), AQP1 (1/800; Abcam, UK), MEF2C (1/800; Abcam, UK), and GAPDH (1/1000; Abcam, UK). After three washes with TBST buffer, the membranes were incubated for $1 \mathrm{~h}$ with appropriate secondary antibody (1/5000; Abcam, UK) at room temperature and visualized by enhanced chemiluminescence (ECL) kit (Beyotime, China). GAPDH was regarded as an internal reference.

\section{Statistical analysis}

The data were presented as mean \pm standard deviation (SD) and analyzed using SPSS 22.0 software (IBM, USA). Differences between two groups were analyzed by student's $t$-test, comparisons between three or more groups were analyzed by one-way analysis of variance; $\mathrm{P}<0.05$ was considered as significant difference.

\section{Results}

\section{MEF 2C is down-regulated in CLP-induced sepsis rats}

In order to characterize the roles of MEF2C in CLP-induced $\mathrm{ALI}$ in rats, the mRNA and protein levels of MEF2C were observed by RT-qPCR and western blot assay, respectively. As shown in Figures $1 \mathrm{~A}$ and $1 \mathrm{~B}$, both mRNA and protein levels of MEF2C were significantly lower in CLP-induced sepsis rats in comparison with the Sham group. These findings concluded that MEF2C was down-regulated in CLP-induced sepsis rats.

\section{Overexpression of MEF2C alleviates lung inflammatory response in CLP-induced sepsis rats}

In order to identify the mechanism of MEF2C in ALI during sepsis, the protective effect of MEF2C overexpression was evaluated in CLP-induced sepsis rats. The CLP rats received intratracheal instillation of AAV-Scramble or AAVMEF2C. The western blot test indicated that AAV-MEF2C treatment significantly promoted MEF2C expression in CLP-induced sepsis rats (Figure 2A). More importantly, CLP challenge induced TNF- $\alpha$, IL-1 $\beta$, IL-6, and IL-10 concentrations in serum in rats at 6 and $12 \mathrm{~h}$, respectively (Figure 2B). Nevertheless, overexpression of MEF2C markedly suppressed TNF- $\alpha$, IL-1 $\beta$, and IL-6 concentrations but promoted IL-10 concentration in serum in CLP-challenged
(A)

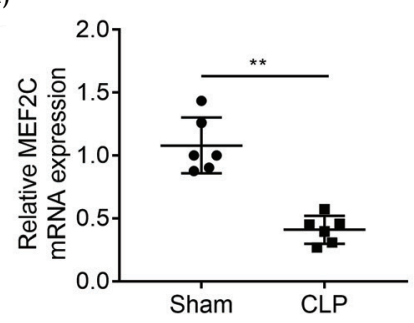

(B)

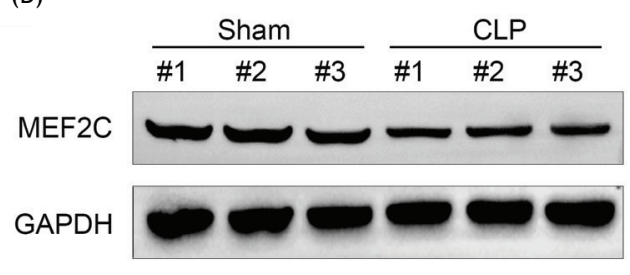

Figure 1 MEF2C is down-regulated in CLP-induced sepsis rats. After the CLP-induced ALI rats model was established, (A) mRNA and (B) protein levels of MEF2C were detected by qRT-PCR and western blot assay; this indicated that MEF2C expression was down-regulated in CLP-induced sepsis rats. Each experiment was repeated thrice. ${ }^{* *} \mathrm{P}<0.01$. 

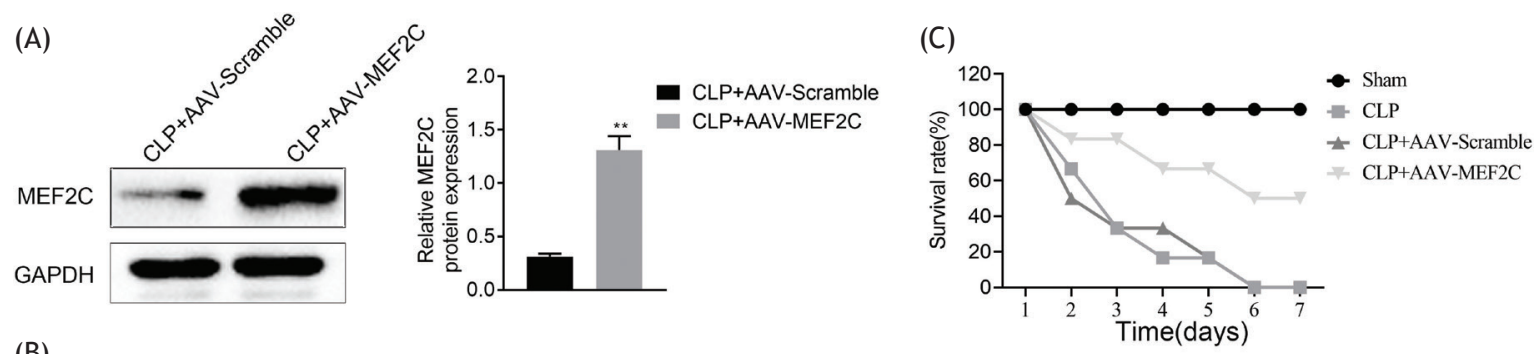

(B)
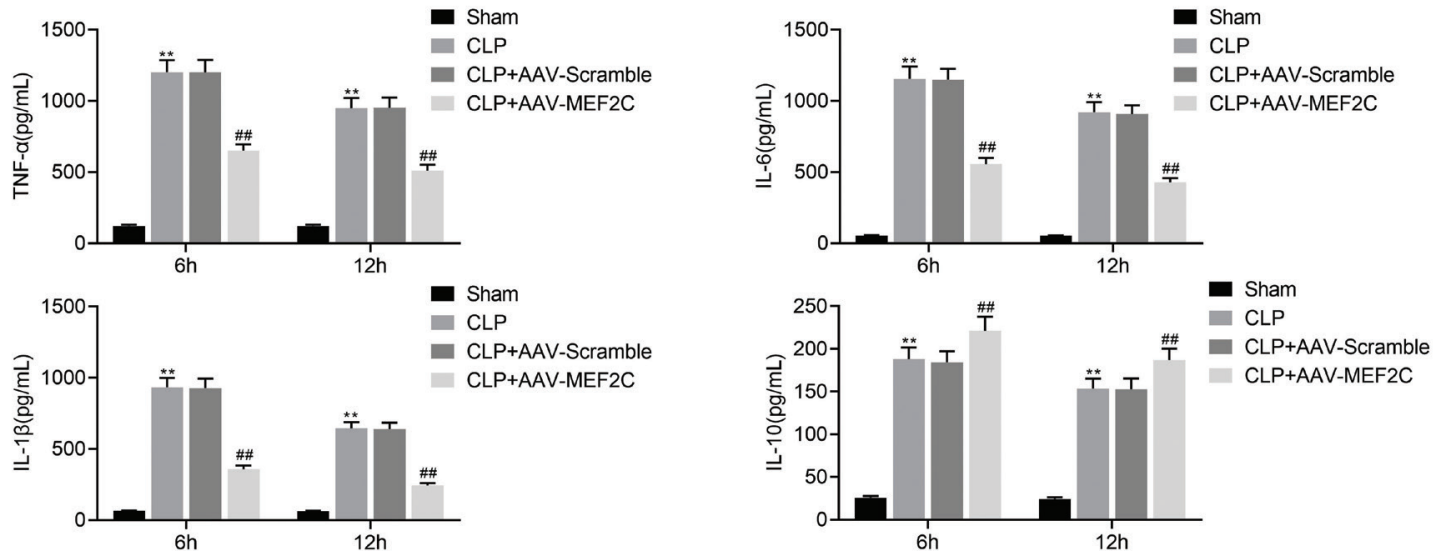

Figure 2 Overexpression of MEF2C alleviates lung inflammatory response in CLP-induced sepsis rats. The CLP rats received intratracheal instillation of AAV-Scramble or AAV-MEF2C. (A) The MEF2C expression was determined by western blot. (B) Inflammation factors, including TNF- $\alpha$, IL-1 $\beta$, IL-6, and IL-10, in serum were examined by commercial ELISA kit at 6 and $12 \mathrm{~h}$. (C) The mortality of rats was recorded. Each experiment was repeated thrice. ${ }^{* *} \mathrm{P}<0.01 \mathrm{vs}$. Sham. ${ }^{\# \# P}<0.01 \mathrm{vs}$. CLP+AAV-Scramble.

rats at 6 and $12 \mathrm{~h}$, thereby indicating that MEF2C alleviated lung inflammatory response in CLP-induced sepsis rats. Furthermore, the mortality of rats was recorded, suggesting that CLP challenge induced rat sacrifices, whereas overexpression of $M E F 2 C$ rescued this phenomenon (Figure 2C).

\section{Overexpression of MEF $2 C$ alleviates CLP-induced lung injury}

In order to investigate the role of IGFBP7 in sepsis-induced $\mathrm{ALI}$, the lung tissues were harvested and subjected to H\&E staining (Figure $3 \mathrm{~A}$ ). There were no obvious histologic changes in the lung tissues of Sham rats. Significant pathologic changes, including mass inflammatory cell infiltration, pulmonary interstitial edema, and alveolar wall thickening, were observed in CLP rats. Fortunately, these changes were alleviated by the overexpression of MEF2C. Additionally, a scoring system was used to assess the degree of lung injury, indicating that CLP surgery increased the lung injury score, which was markedly decreased by the overexpression of MEF2C. The wet-dry weight ratio of the lungs is an important characteristic of $\mathrm{ALI}$ and a commonly used indicator of pulmonary vascular permeability. CLP-challenged rats showed a significant increase in the lung wet-dry weight ratio compared to Sham rats at 12 and $24 \mathrm{~h}$, respectively, which was decreased by the overexpression of MEF2C (Figure $3 B)$. Moreover, compared to Sham rats, CLP surgery promoted serum lactate dehydrogenase (LDH) concentration in lung tissues, which was reversed by the overexpression of MEF2C (Figure 3C). The MPO activity in lung tissues was measured, revealing that CLP challenge enhanced MPO activity at 12 and $24 \mathrm{~h}$, which was reduced by the overexpression of MEF2C (Figure 3D). Therefore, these findings demonstrated that overexpression of MEF2C alleviated CLP-induced lung injury.

\section{Overexpression of MEF $2 C$ suppresses apoptosis in CLP-induced sepsis rats}

To explore mechanism underlying the protective effects of MEF2C on sepsis-induced ALI, cell apoptosis in the lungs of CLP-induced sepsis rats was determined by TUNEL staining. The lung tissue sections from CLP rats accompanied with an increased number of TUNEL-positive cells, which was reduced by the overexpression of MEF2C (Figure 4A). Moreover, the western blot examination suggested that BAX expression was promoted and Bcl-2 expression was suppressed by CLP challenge in comparison to Sham rats, while overexpression of MEF2C led to a significant decrease of $\mathrm{BAX}$ and an increase of Bcl-2 (Figure 4B). Thus, these findings concluded that overexpression of MEF2C suppressed apoptosis in CLP-induced sepsis rats.

\section{MEF 2C alleviates CLP-induced lung inflammatory response and apoptosis via up-regulating AQP1}

In clinic, promising drug targets may depict aquaporins (AQPs) as they modulate crucial mechanisms in sepsis. By examining AQP1 expression, we demonstrated that CLP challenge reduced both mRNA and protein levels of AQP1 in rats, whereas they were promoted by the overexpression 
(A)

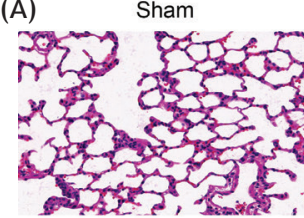
CLP+AAV-Scramble

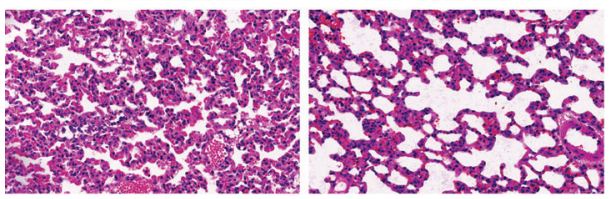

CLP

CLP+AAV-MEF2C

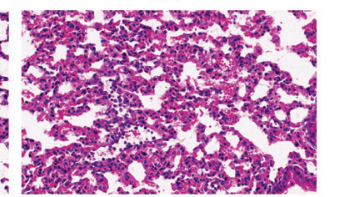

(C)
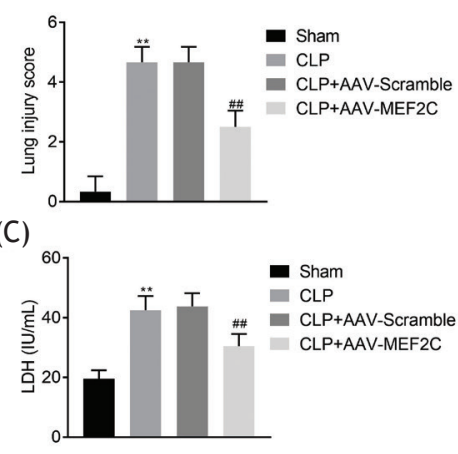

(B)

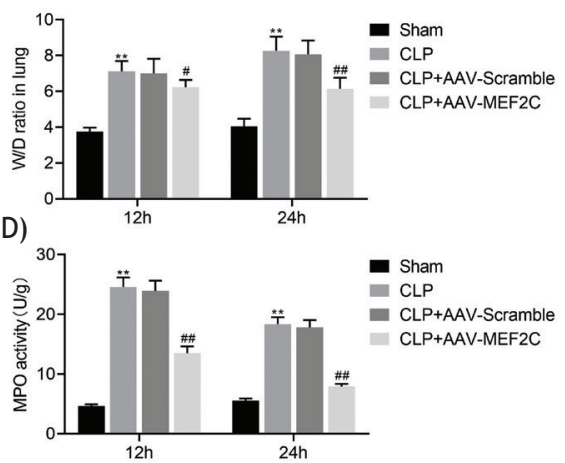

Figure 3 Overexpression of MEF2C alleviates CLP-induced lung injury. (A) Representative histology sections of the lung tissues under light microscope $(\mathrm{HEE}$ staining $\times 200)$. In addition, the lung histologic injury score was calculated. (B) Wet/dry ratio was measured at 12 and $24 \mathrm{~h}$, respectively. (C) LDH concentration in the lung tissues was measured by commercial ELISA kit. (D) MPO activity in the lung tissues was measured at 12 and $24 \mathrm{~h}$ after CLP challenge. Each experiment was repeated thrice. ${ }^{* *} \mathrm{P}<0.01$ vs. Sham. \#P $<0.01$ vs. CLP+AAV-Scramble.

(A)

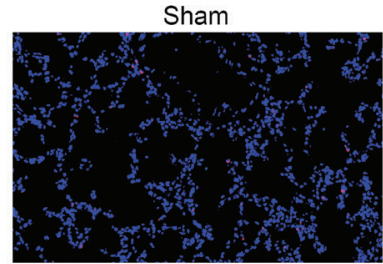

CLP

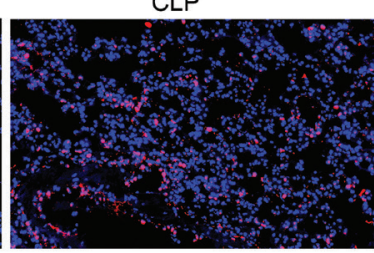

CLP+AAV-Scramble

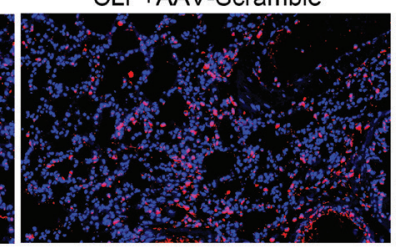

CLP+AAV-MEF2C

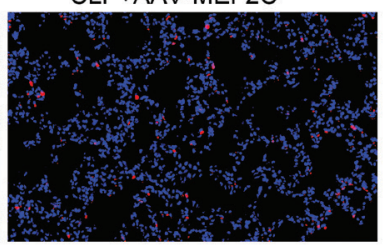

TUNEL DAPI

(B)
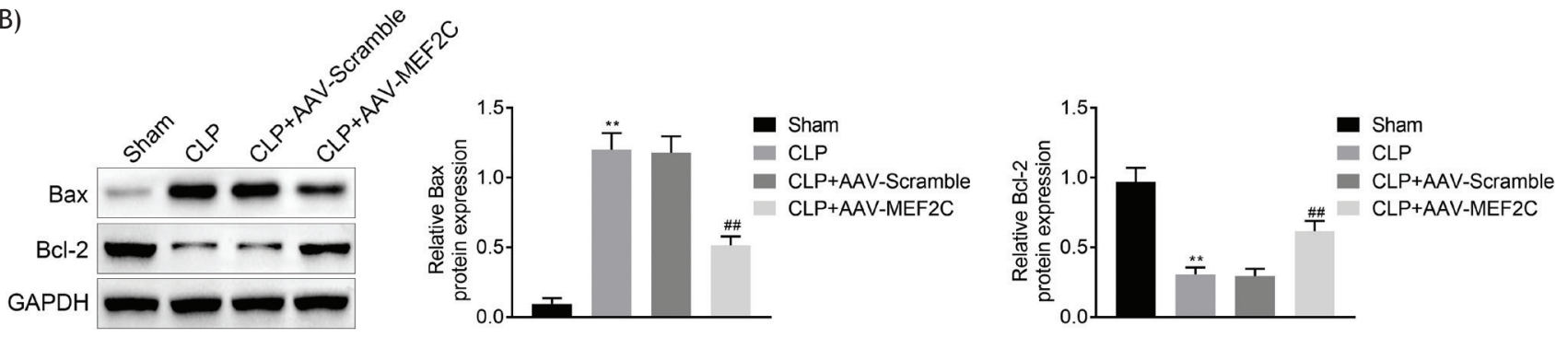

Figure 4 Overexpression of MEF2C suppresses apoptosis in CLP-induced sepsis rats. (A) Cell apoptosis in the lung tissues was determined by TUNEL staining assay. (B) BAX and Bcl-2 expressions were detected by western blot assay. Each experiment was repeated thrice. ${ }^{* *} \mathrm{P}<0.01$ vs. Sham. ${ }^{\# \#} \mathrm{P}<0.01$ vs. CLP+AAV-Scramble.

of MEF2C (Figures 5A and B). Interestingly, by knockdown of AQP1 in CLP rats, the MEF2C-induced decrease of BAX and increase of $\mathrm{BCl}-2$ were reversed by sh-AQP1, indicating that knockdown of AQP1 promoted cell apoptosis (Figure $5 C)$. Besides, MEF2C declined TNF- $\alpha$, IL-1 $\beta$, and IL- 6 concentrations but promoted IL-10 concentration in serum, which was reversed by knockdown of AQP1 (Figure 5D). Therefore, these findings confirmed that MEF2C alleviated CLP-induced lung inflammatory response and apoptosis via up-regulating AQP1.

\section{Discussion}

In this study, the CLP-induced sepsis rat model was established, and MEF2C was down-regulated in this model. Moreover, AAV-MEF2C treatment markedly suppressed
TNF- $\alpha$, IL-1 $\beta$, and IL-6 concentrations but promoted $\mathrm{IL}-10$ concentration in the serum of CLP-challenged rats. Besides, overexpression of MEF2C alleviated CLP-induced lung injury. Interestingly, AAV-MEF2C treatment was confirmed to suppress apoptosis in CLP-induced sepsis rats, and promoted APQ1 expression. Mechanistically, the rescue experiments indicated that MEF2C alleviated CLP-induced lung inflammatory response and apoptosis via up-regulating AQP1. Taken together, our study demonstrated that overexpression of MEF2C suppressed CLP-induced lung inflammatory response and apoptosis via up-regulating AQP1, providing a novel therapeutic target for sepsis-induced ALI.

The essence of ALI is believed to be an excessive, uncontrolled inflammatory response. Extensive pulmonary inflammation contributes to the destruction of the basement membrane and increases the permeability of the alveolar-capillary membrane..$^{13}$ Release of pro-inflammatory 
(A)

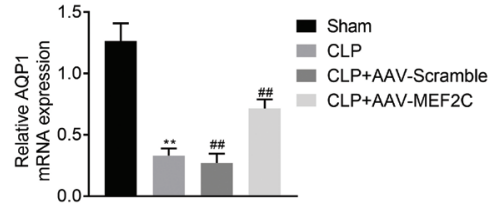

(B)

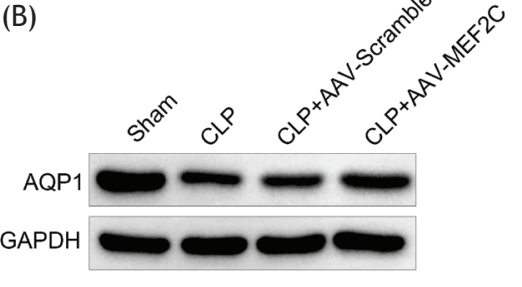

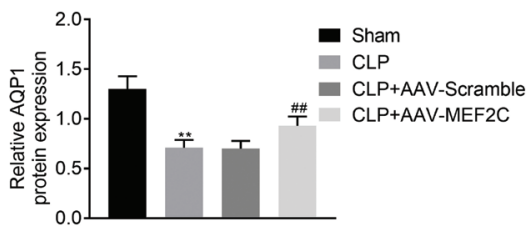

(C)
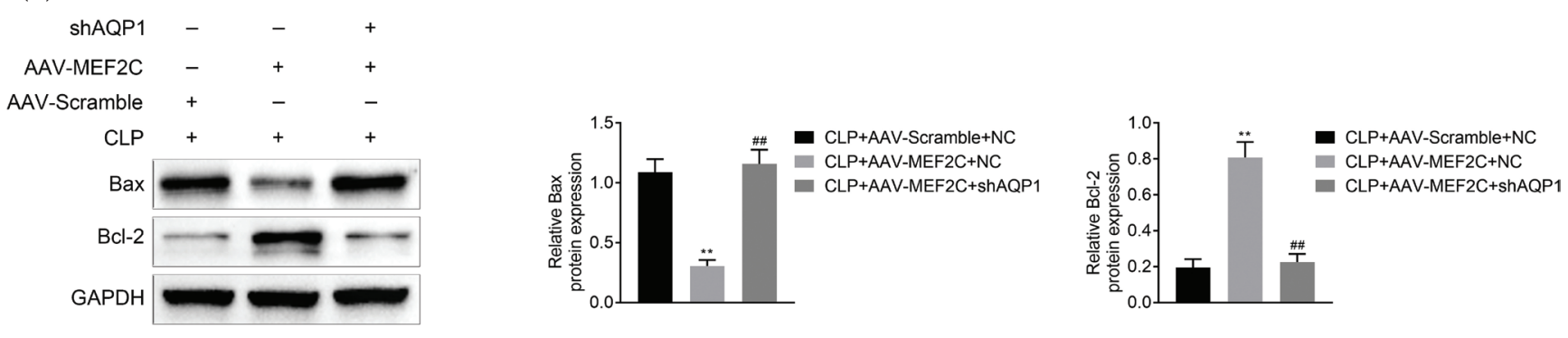

(D)
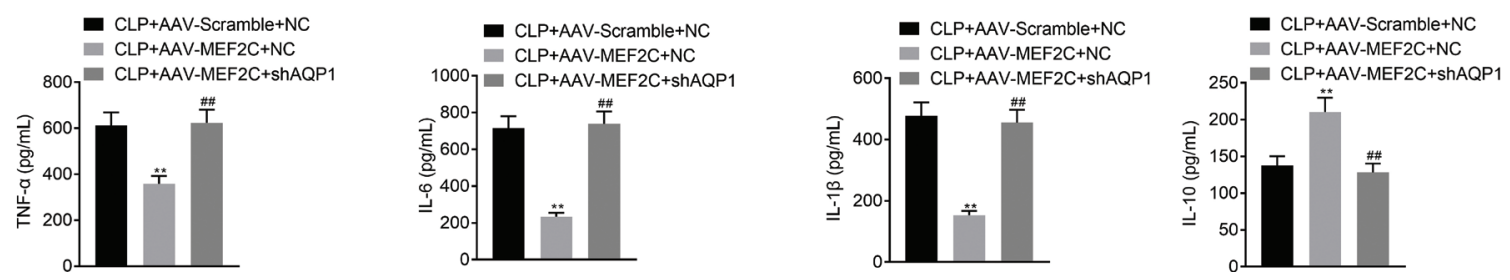

Figure 5 MEF2C alleviates CLP-induced lung inflammatory response and apoptosis via up-regulating AQP1. After transfected with sh-AQP1 or sh-NC, the rats were treated with AAV-MEF2C. (A) mRNA and (B) protein levels of AQP1 were detected by RT-qPCR and western blot assay, respectively. (C) Expressions of Bcl-2 and BAX were determined by western blot assay. (D) Concentrations of inflammatory cytokines, including TNF- $\alpha$, IL-1 $\beta$, IL-6, and IL-10, in serum were evaluated by commercial ELISA kit. Each experiment

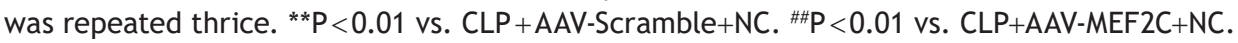

mediators, such as TNF- $\alpha$, IL-1 $\beta$, and IL-6, has been shown to play a key role in the early phase of ALI. ${ }^{14}$ Similarly, production of TNF- $\alpha$, IL-1 $\beta$, IL- 6 , and IL-10 was markedly increased by CLP challenge. Accumulating evidence has confirmed that suppression of inflammatory responses efficiently protects against sepsis-induced lung injury. Isoliquiritigenin alleviates sepsis-induced lung injury through suppressing inflammatory responses..$^{15}$ Ghrelin exerts a protective role against sepsis-induced ALI, probably by reducing the production of inflammatory cytokines. ${ }^{16}$ In the present study, increase in TNF- $\alpha$, IL-1 $\beta$, and IL- 6 production was alleviated by the overexpression of MEF2C. Nevertheless, production of IL-10 was further increased by the overexpression of MEF2C. This phenomenon occurs because IL-10 is shown to mediate protection in sepsis. ${ }^{17}$ Meanwhile, these findings suggested the anti-inflammatory effect of MEF2C in vivo.

Dysfunctioning of inflammatory response leads to uncontrolled inflammatory response and subsequent immunosuppression. ${ }^{18}$ Uncontrolled apoptosis is thought to be the main cause of significant immunosuppression..$^{19}$ Hence, dysregulation of apoptosis occurs in immune and nonimmune cells in sepsis. ${ }^{20}$ As a novel therapeutic strategy, it has been reported that methane alleviates sepsis-induced injury by inhibiting apoptosis in vivo and in vitro. ${ }^{21}$ Jiang et al. have confirmed that circC3P1 attenuates pro-inflammatory cytokine production and cell apoptosis in sepsis-induced ALI by modulating miR-21.22 During sepsis, increased numbers of TUNEL-positive cells in the lung sections were observed. ${ }^{23}$ Currently, we demonstrated that CLP challenge elevated the number of TUNEL-positive cells in the lung section, which was further reduced by the overexpression of MEF2C, suggesting the anti-apoptosis effect of MEF2C in vivo.

Aquaporin AQP1 is primarily expressed in the lung. ${ }^{24}$ Nevertheless, after lipopolysaccharide (LPS) exposure, AQP1 expression is decreased in rat lungs. ${ }^{25} \mathrm{As}$ a therapeutic option, hydrogen-rich saline and parenteral vitamin C can protect against sepsis-induced lung injury by attenuating AQP1 expression. ${ }^{26,27}$ In addition, AQP1 expression is declined in the lung after inflammatory stimulation, but up-regulated AQP1 attenuates lung inflammation. ${ }^{10,26}$ In line with this result, we demonstrated that knockdown of AQP1 significantly promoted the production of TNF- $\alpha, \mathrm{IL}-1 \beta$, and IL- 6 but suppressed the IL-10 production. If treated with emodin, the up-regulation of AQP1 reduces the inflammatory cytokine release and pulmonary apoptosis in lung sepsis, suggesting that emodin suppresses inflammation, leading a lower mortality in CLP-induced ALI. ${ }^{28}$ Meanwhile, AQP1 is identified as a pathway in the hyperoxia-induced lung injury. ${ }^{29}$ In vitro, CASC2/miR-144-3p/AQP1 axis suppresses LPS-induced A549 cell apoptosis. ${ }^{30}$ Consistent with these findings, APQ1 was promoted by MER2C and then 
suppressed cell apoptosis. These findings concluded that the anti-inflammatory and anti-apoptotic effects of MEF2C were functioned through up-regulation of AQP1. More importantly, AQP-1 is involved in sodium and water homeostasis. When inflammatory mediators down-regulate AQP-1 protein expression, removal of pulmonary edema fluid is impeded, revealing that down-regulation of AQP-1 induces pulmonary edema. ${ }^{31}$ Thus, the MEF $2 \mathrm{C}$ could promote AQP-1 expression via reducing levels of inflammatory mediators and subsequently inducing removal of pulmonary edema fluid, thereby alleviating ALI.

The present study has certain limitations. It has been reported that MEF2C cooperates with $\mathrm{Sp} 1$ to activate human AQP1 transcription by binding to its promoter in HUVEC cells. ${ }^{32}$ Nevertheless, the delineation of transcriptional regulation of $A Q P 1$ by $M E F 2 C$ in sepsis remains unclear. Second, NF- $\kappa B$ is a vital signal pathway in sepsis. ${ }^{33}$ More importantly, transcription factor MEF2C inhibited endothelial cell inflammation via modulating $N F-\kappa B .^{7}$ The relationship between $M E F 2 C$ and $N F-\kappa B$ signal pathway remains unclear. These investigations are not expounded amply and require further exploration.

\section{Conclusion}

The current study demonstrated that MEF2C expression was enhanced in CLP-induced sepsis rats. Overexpression of MEF2C could alleviate $\mathrm{ALI}$, and reduce lung inflammatory response and apoptosis. MEF2C alleviated CLP-induced lung inflammatory response and apoptosis via up-regulating AQP1. As such, agent-facilitating MEF2C expression could be a promising strategy for preventing sepsis-induced ALI.

\section{Funding}

This work was supported by the National Natural Science Foundation of China (grant No. 81960362).

\section{Competing interests}

The authors declare that there are no conflicts of interest to disclose.

\section{Contribution of authors}

Wenmei Liang and Li Guo designed the study, supervised the data collection. Tonghua Liu analyzed and interpreted the data. Song Qin prepared and reviewed draft of the manuscript for publication. All authors read and approved the final manuscript.

\section{References}

1. Seckel MA, Ahrens T. Challenges in sepsis care: New sepsis definitions and fluid resuscitation beyond the central venous pressure. Crit Care Nurs Clin North Am. 2016;28(4):513-32. https://doi.org/10.1016/j.cnc.2016.08.001
2. Kaplan MY, Ozen S, Akcal O, Gulez N, Genel F. Autoimmune and inflammatory manifestations in pediatric patients with primary immunodeficiencies and their importance as a warning sign. Allergologia et Immunopathol. 2020;48(6):701-10. https://doi.org/10.1016/j.aller.2020.02.009

3. Olvera L, Dutra D. Early recognition and management of maternal sepsis. Nurs Women's Health. 2016;20(2):182-95; quiz 96. https://doi.org/10.1016/j.nwh.2016.02.003.

4. Han IM, Yoon CY, Shin DH, Kee YK, Han SG, Kwon YE, et al. Delta neutrophil index is an independent predictor of mortality in septic acute kidney injury patients treated with continuous renal replacement therapy. BMC Nephrol. 2017;18(1):94. https://doi.org/10.1186/s12882-017-0507-8

5. Li R, Ren T, Zeng J. Mitochondrial coenzyme $Q$ protects sepsis-induced acute lung injury by activating PI3K/ Akt/GSK-3beta/mTOR pathway in rats. BioMed Res Int. 2019;2019:5240898. https://doi.org/10.1155/2019/5240898

6. Deczkowska A, Matcovitch-Natan O, Tsitsou-Kampeli A, Ben-Hamo S, Dvir-Szternfeld R, Spinrad A, et al. Mef2C restrains microglial inflammatory response and is lost in brain ageing in an IFN-I-dependent manner. Nat Commun. 2017;8(1):717. https://doi.org/10.1038/s41467-017-00769-0

7. Xu Z, Yoshida T, Wu L, Maiti D, Cebotaru L, Duh EJ. Transcription factor MEF2C suppresses endothelial cell inflammation via regulation of NF-kappaB and KLF2. J Cell Physiol. 2015;230(6):1310-20. https://doi.org/10.1002/jcp.24870

8. Sahoo S, Meijles DN, Al Ghouleh I, Tandon M, CifuentesPagano E, Sembrat J, et al. MEF2C-MYOCD and Leiomodin1 suppression by miRNA-214 promotes smooth muscle cell phenotype switching in pulmonary arterial hypertension. PloS One. 2016;11(5):e0153780. https://doi.org/10.1371/journal. pone. 0153780.

9. Hua Y, Ying X, Qian Y, Liu H, Lan Y, Xie A, et al. Physiological and pathological impact of AQP1 knockout in mice. Biosci Rep. 2019 May;39(5):BSR20182303. https://doi.org/10.1042/ BSR20182303

10. Kang X, Lu XG, Zhan LB, Liang ZK, Guo WX, Ma Q, et al. DaiHuang-Fu-Zi-Tang alleviates pulmonary and intestinal injury with severe acute pancreatitis via regulating aquaporins in rats. BMC Compl Altern Med. 2017;17(1):288. https://doi. org/10.1186/s12906-017-1789-x

11. Gao Z, Xu J, Sun D, Zhang R, Liang R, Wang L, et al. Traditional Chinese medicine, Qing Ying Tang, ameliorates the severity of acute lung injury induced by severe acute pancreatitis in rats via the upregulation of aquaporin-1. Exp Ther Med. 2014;8(6):1819-24. https://doi.org/10.3892/etm.2014.1987

12. Jiang Y, Ma R, Zhao Y, Li GJ, Wang AK, Lin WL, et al. MEF2C/ miR-133a-3p.1 circuit-stabilized AQP1 expression maintains endothelial water homeostasis. FEBS Lett. 2019;593(18):256673. https://doi.org/10.1002/1873-3468.13516

13. Williams AE, Chambers RC. The mercurial nature of neutrophils: Still an enigma in ARDS? Am J Physiol Lung Cell Mol Physiol. 2014;306(3):L217-30. https://doi.org/10.1152/ ajplung.00311.2013

14. Xia W, Pan Z, Zhang H, Zhou Q, Liu Y. Inhibition of ERRalpha aggravates sepsis-induced acute lung injury in rats via provoking inflammation and oxidative stress. Oxid Med Cell Long. 2020;2020:2048632. https://doi. org $/ 10.1155 / 2020 / 2048632$

15. Chen X, Cai X, Le R, Zhang M, Gu X, Shen F, et al. Isoliquiritigenin protects against sepsis-induced lung and liver injury by reducing inflammatory responses. Biochem Biophy Res Comm. 2018;496(2):245-52. https://doi.org/10.1016/j.bbrc.2017.11.159

16. Zheng $\mathrm{H}$, Liang $\mathrm{W}$, He W, Huang $\mathrm{C}$, Chen Q, Yi H, et al. Ghrelin attenuates sepsis-induced acute lung injury by inhibiting the NF-kappaB, iNOS, and Akt signaling in alveolar macrophages. Am J Physiol Lung Cell Mol Physiol. 2019;317(3):L381-91. https://doi.org/10.1152/ajplung.00253.2018 
17. Park KS, Svennerholm K, Shelke GV, Bandeira E, Lasser C, Jang SC, et al. Mesenchymal stromal cell-derived nanovesicles ameliorate bacterial outer membrane vesicle-induced sepsis via IL-10. Stem Cell Res Ther. 2019;10(1):231. https://doi. org/10.1186/s13287-019-1352-4

18. Girardot T, Rimmele T, Venet F, Monneret G. Apoptosisinduced lymphopenia in sepsis and other severe injuries. Apoptosis Int J Prog Cell Death. 2017;22(2):295-305. https:// doi.org/10.1007/s10495-016-1325-3

19. Cao C, Yu M, Chai Y. Pathological alteration and therapeutic implications of sepsis-induced immune cell apoptosis. Cell Death Dis. 2019;10(10):782. https://doi.org/10.1038/ s41419-019-2015-1

20. Mahidhara R, Billiar TR. Apoptosis in sepsis. Crit Care Med. 2000;28(4 Suppl):N105-13. https://doi.org/10.1097/00003246200004001-00013.

21. Li Z, Jia $Y$, Feng $Y$, Cui R, Miao R, Zhang $X$, et al. Methane alleviates sepsis-induced injury by inhibiting pyroptosis and apoptosis: In vivo and in vitro experiments. Aging. 2019;11(4):1226-39. https://doi.org/10.18632/aging.101831

22. Jiang WY, Ren J, Zhang XH, Lu ZL, Feng HJ, Yao XL, et al. CircC3P1 attenuated pro-inflammatory cytokine production and cell apoptosis in acute lung injury induced by sepsis through modulating miR-21. J Cell Mol Med. 2020;24(19):112219. https://doi.org/10.1111/jcmm.15685

23. Chopra M, Reuben JS, Sharma AC. Acute lung injury: Apoptosis and signaling mechanisms. Exp Biol Med. 2009;234(4):361-71. https://doi.org/10.3181/0811-MR-318

24. Hong-Min F, Chun-Rong H, Rui Z, Li-Na S, Ya-Jun W, Li L. CGRP 8-37 enhances lipopolysaccharide-induced acute lung injury and regulating aquaporin 1 and 5 expressions in rats. J Physiol Biochem. 2016;73(3):381-6. https://doi.org/10.1007/ s13105-017-0563-3

25. Ma T, Liu Z. Functions of aquaporin 1 and alpha-epithelial $\mathrm{Na}+$ channel in rat acute lung injury induced by acute ischemic kidney injury. Int Urol Nephrol. 2013;45(4):1187-96. https:// doi.org/10.1007/s11255-012-0355-1

26. Tao B, Liu L, Wang N, Wang W, Jiang J, Zhang J. Effects of hydrogen-rich saline on aquaporin 1,5 in septic rat lungs. J Surg Res. 2016;202(2):291-8. https://doi.org/10.1016/j. jss.2016.01.009

27. Fisher BJ, Kraskauskas D, Martin EJ, Farkas D, Wegelin JA, Brophy D, et al. Mechanisms of attenuation of abdominal sepsis induced acute lung injury by ascorbic acid. Am J Physiol Lung Cell Mol Physiol. 2012;303(1):L20-32. https://doi. org/10.1152/ajplung.00300.2011.

28. Guo R, Li Y, Han M, Liu J, Sun Y. Emodin attenuates acute lung injury in cecal-ligation and puncture rats. Int Immunopharm. 2020;85:106626. https://doi.org/10.1016/j.intimp.2020.106626

29. Liu D, Wang Y, Li L, Zhao H, Li L, Liu Y, et al. Celecoxib protects hyperoxia-induced lung injury via NF-kappaB and AQP1. Front Pediatr. 2019;7:228. https://doi.org/10.3389/fped.2019.00228

30. Li H, Shi H, Gao M, Ma N, Sun R. Long non-coding RNA CASC2 improved acute lung injury by regulating miR-144-3p/AQP1 axis to reduce lung epithelial cell apoptosis. Cell Biosci. 2018;8:15. https://doi.org/10.1186/s13578-018-0205-7

31. Ma C, Dong L, Li M, Cai W. Qidonghuoxue decoction ameliorates pulmonary edema in acute lung injury mice through the upregulation of epithelial sodium channel and aquaporin-1. Evidence Complement Altern Med (eCAM). 2020;2020:2492304. https://doi.org/10.1155/2020/2492304

32. Jiang $\mathrm{Y}$, Liu H, Liu WJ, Tong HB, Chen CJ, Lin FG, et al. Endothelial aquaporin-1 (AQP1) expression is regulated by transcription factor Mef2c. Mol Cells. 2016;39(4):292-8. https://doi.org/10.14348/molcells.2016.2223

33. Li X, Su J, Cui X, Li Y, Barochia A, Eichacker PQ. Can we predict the effects of NF-kappaB inhibition in sepsis? Studies with parthenolide and ethyl pyruvate. Expert Opin Invest Drugs. 2009;18(8):1047-60. https://doi.org/10.1517/ 13543780903018880 